\title{
Does the addition of hip strengthening exercises improve outcomes following total knee arthroplasty? A study protocol for a randomized trial
}

\author{
Margaret B. Schache ${ }^{1,2^{*}}$, Jodie A. McClelland ${ }^{1}$ and Kate E. Webster ${ }^{1}$
}

\begin{abstract}
Background: Total knee arthroplasty (TKA) is effective in reducing pain and improving function for end-stage knee osteoarthritis. However, muscle weakness and functional limitations persist despite assistance from post-operative rehabilitation programs that traditionally focus on quadriceps strengthening and range of movement exercises. Hip abductor muscle weakness is evident in knee osteoarthritis and hip muscle strengthening reduces knee pain in this group. Following TKA, people with weak hip abductor strength perform more poorly on measures of physical function. However, very little is known of the effectiveness of including hip abductor strengthening exercises in post-operative rehabilitation. The aim of this trial is to compare the effects of targeted hip abductor strengthening to those of traditional care in a TKA rehabilitation program on muscle strength, patient reported outcomes and functional performance measures.
\end{abstract}

Methods/design: This protocol describes a single-blinded randomized controlled trial, where 104 participants referred for inpatient rehabilitation following TKA will be recruited. Participants will be randomized using computer-generated numbers to one of two groups: usual care or usual care with additional hip strengthening exercises. Participants will attend physiotherapy daily during their inpatient length of stay, and will then attend between six and eight physiotherapy sessions as an outpatient. Primary outcomes are isometric hip abductor strength and the Knee Injury and Osteoarthritis Outcome Score (KOOS). Secondary outcomes are stair climb test, 6 min walk test, timed up and go, 40 m fast-paced walk test, 30 second chair stand test, isometric quadriceps strength, Lower Extremity Functional Scale (LEFS) and SF-12. Outcome measures will be recorded at baseline (admission to inpatient rehabilitation), and then 3 weeks, 6 weeks and 6 months post admission to rehabilitation.

Discussion: The findings of this study will determine whether the addition of targeted hip strengthening to usual care rehabilitation improves physical performance and patient reported outcomes following TKA when compared to usual care rehabilitation. This will then determine whether targeted hip strengthening exercises should be included in traditional rehabilitation programs to improve the outcomes following total knee arthroplasty.

Trial registration: The trial protocol was registered with the Australian Clinical Trial Registry (ACTRN12615000863538) on 18 August 2015.

Keywords: Total knee arthroplasty, Hip strengthening, Randomized controlled trial, Exercise, Rehabilitation

\footnotetext{
* Correspondence: schachem@ramsayhealth.com.au

${ }^{1}$ School of Allied Health, La Trobe University, Melbourne, Australia

${ }^{2}$ Physiotherapy Department, Donvale Rehabilitation Hospital, Ramsay Health

Care, 1119 Doncaster Rd, Donvale, Melbourne 3111, Australia
} 


\section{Background}

End-stage knee osteoarthritis (OA) is a significant health issue worldwide resulting in severe pain and disability $[1,2]$. Total knee arthoplasty (TKA) leads to significant improvements in pain and the performance of functional activities such as walking for patients with end-stage knee OA [3]. Improvements in pain and functional performance are achieved with assistance from rehabilitation programs [3-6]. Traditional rehabilitation programs typically focus on improving knee strength and range of movement, and improving gait and stair climbing [7-10]. For most programs, emphasis is placed on achieving optimal knee range of movement and improving quadriceps muscle strength [11].

Despite completing traditional rehabilitation programs, functional limitations persist in patients following TKA compared to healthy age-matched individuals. Following TKA, people have reduced walking speed, and report difficulty climbing stairs and rising from a chair $[12,13]$. They also perform more poorly on tests of Timed Up and Go (TUG) and stair climbing times than healthy adults $[3,13]$. The persistence of functional limitations demonstrates that there is a need to expand on the focus of current rehabilitation practices to restore the function of patients following TKA to the levels of healthy adults.

Prior to surgery, patients with end-stage knee OA demonstrate reduced hip abductor strength [14]. They exhibit altered gait patterns that may be an attempt to avoid knee pain, to minimize forces through the affected cartilage, or to reduce the sensation of the knee giving way [15]. Consequently, patients with severe knee OA walk with decreased gait speed, reduced stride length, and increased time in double limb support [16]. These factors combined with reduced activity may result in lowered activation and diminished strength of the hip abductors over a period of time [17]. There has been a small quantity of research with promising findings that targeted hip strengthening programs in patients with end stage knee OA may lead to improvement in symptoms and quality of life $[18,19]$.

Although often ignored in post-operative rehabilitation programs, there is sufficient evidence that the hip abductor weakness present prior to surgery continues after TKA and is not improved with current rehabilitation interventions [20, 21]. Post-operative pain, reduced demands on the operated limb in the early post-operative period and habitual gait patterns are also likely to contribute to further reductions in hip abductor strength. Following TKA, there is a correlation between hip abductor strength and functional outcomes [20,21]. Hip abductor strength contributes to physical function such as turning whilst walking and rising from a chair in people with unilateral TKA. There is a correlation between stronger hip abductor muscles and faster times for TUG and stair climb tests [20, 21]. Unless rehabilitation programs specifically include hip abductor strengthening exercises, it is unlikely that the hip abductors will return to normal levels of strength, contributing to ongoing difficulties in activities of daily living such as walking and stair climbing. Given the improvement in strength seen in pre-operative patients, it is likely that similar rehabilitation efforts will yield improvements in function post-operatively. Despite this, no study has investigated the effects of hip abductor strengthening on improving strength or function following TKA.

The aim of this randomized controlled trial (RCT) is to compare the effects of targeted hip abductor strengthening with those of traditional care in a TKA rehabilitation program on muscle strength, patient reported outcomes and functional performance measures.

\section{Methods/design \\ Trial design}

The study design is a single-blinded randomized controlled trial (Fig. 1) adhering to Standard Protocol Items: Recommendation for Interventional Trials (SPIRIT) guidelines [22]. Outcomes will be measured at admission to inpatient rehabilitation, and at 3 weeks, 6 weeks and 6 months following the commencement of rehabilitation. These time points were chosen to adequately measure the rate of improvement in all outcome measures.

The trial protocol was registered with the Australian New Zealand Clinical Trials Registry (ANZCTR number: 12615000863538) on 18 August 2015. Ethical approval was granted by La Trobe University Faculty Human Ethics Committee (FHEC 14/256). Reporting of the RCT will follow the CONSORT guidelines for randomized trials of nonpharmacologic treatment (Fig. 1) [23].

\section{Participants}

A sample of 104 males and females with primary unilateral TKA will be recruited between October 2015 and March 2017 from a 90 bed private rehabilitation hospital that treats patients following joint replacements, multitrauma, stroke, cardiac surgery and general deconditioning.

Participants will be eligible if they are aged 50 years or older, have had a primary unilateral TKA for end-stage knee osteoarthritis in the previous two weeks and are admitted for inpatient rehabilitation followed by outpatient rehabilitation.

Participants will be excluded in the presence of unstable medical conditions preventing the patient from participating in the rehabilitation programs, history of ipsilateral hip replacement, ipsilateral hip osteoarthritis or lateral hip pain, neurological or any other conditions affecting strength or function of lower limbs. Eligibility of prospective patients will be identified on their admission to 


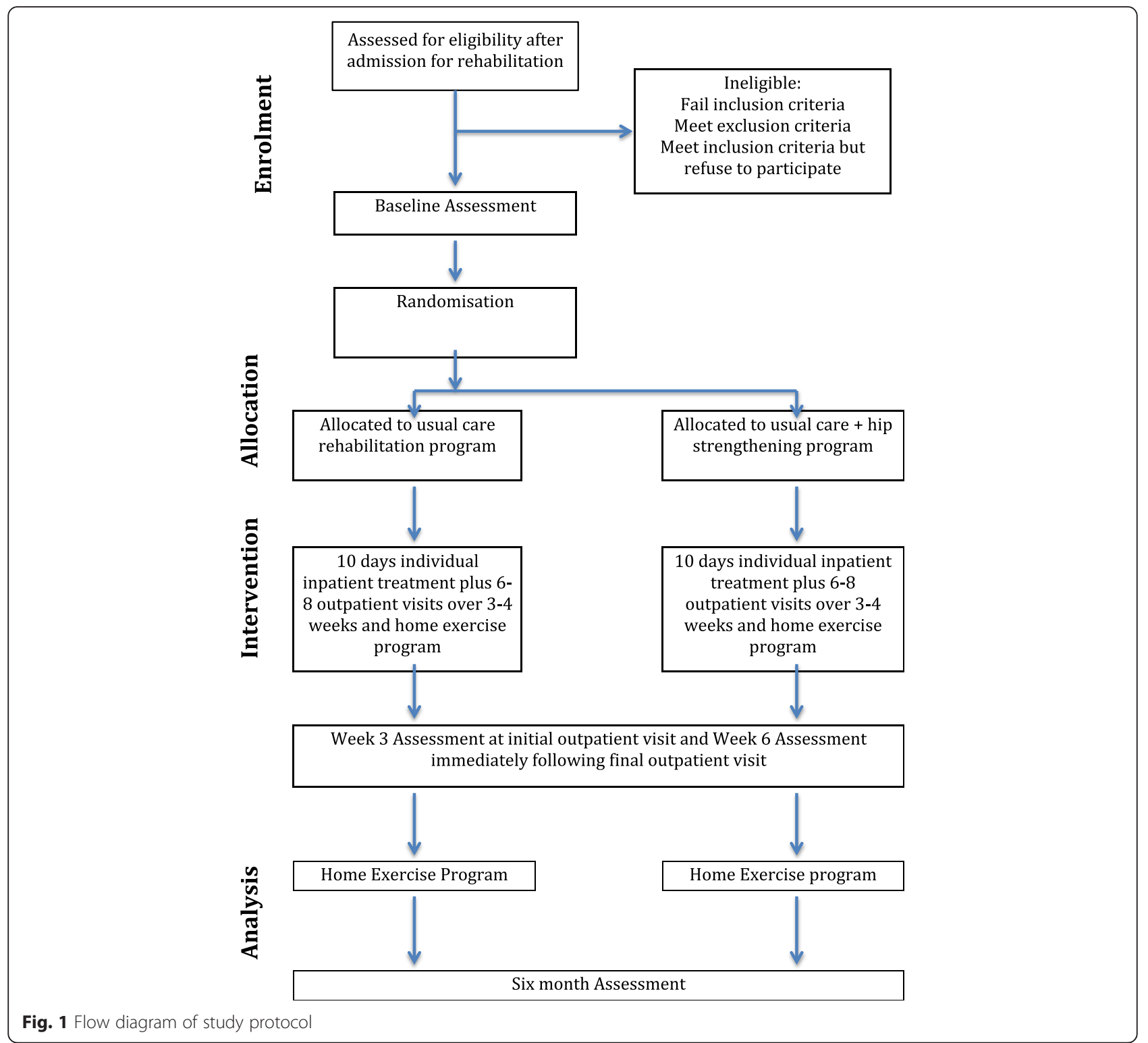

inpatient rehabilitation from the acute hospitals by the treating physiotherapists.

\section{Randomisation and allocation concealment}

Eligible patients will be identified by the treating physiotherapist. The treating physiotherapist will inform the patient of the study, give them the patient information sheet and arrange for a meeting with the primary researcher. The primary researcher will discuss any questions with the patient and gain informed consent of willing participants. Eligible patients will be randomized by a computer-generated list of random numbers to usual care or usual care plus hip strengthening. An independent person, not involved in delivering the intervention or testing, will manage the allocation list and numbered opaque envelopes containing the allocated group of each participant. Only the treating physiotherapist will be informed of the group allocation of the participants. The allocation list will not be available to the researchers and the participants will not be informed which group they are in. The participants will be informed that there are two different rehabilitation programs but will not be told that one program is specifically targeting hip strengthening exercises. This is to prevent potential bias from knowledge of treatment. The rehabilitation will be conducted in a large busy gym where there are many different patients with different diagnoses treated at the same time by their individual physiotherapists with individual exercise programs dependent on each patient's needs. This will reduce the probability of patients from one intervention seeing the other intervention group's exercises. A survey on exit from the study will be conducted 
to determine which group the participants thought they were in during their rehabilitation.

\section{Procedure}

Baseline and all follow-up assessments will be performed in the physiotherapy department at the rehabilitation hospital by a blinded assessor. The assessments of isometric muscle strength, knee range of movement, step tap test and 30-second chair stand test will be conducted in a closed private treatment room in the physiotherapy department. The Timed Up and Go Test and Stair Climb Test will be assessed in a corner of the gym away from the patient treatment area. The 6-minute walk and $40 \mathrm{~m}$ Fast-paced Walk will be assessed along a premeasured $50 \mathrm{~m}$-long walkway located on the other side of the hospital. This will prevent the assessor from observing the interventions and the treating physiotherapists observing the assessments. All participants will attend daily physiotherapy for their length of stay as an inpatient, and will then attend between six and eight physiotherapy sessions as an outpatient. All participants will be given a home exercise program on discharge from inpatient rehabilitation. All participants will attend weekly outpatient physiotherapy sessions and during these sessions, the home exercise program will be progressed.

\section{Interventions}

\section{Usual care}

All participants in this trial will participate in the usual care structure of rehabilitation at the treating institution. Under the usual care rehabilitation, inpatients at the hospital with TKA attend either two 45-min sessions of physiotherapy or one 45-min session of physiotherapy and one 45-min session of hydrotherapy every day on five days of the week. All participants also attend one 45-min session of either physiotherapy or hydrotherapy on the other two weekend days. All patients at the rehabilitation hospital receive hydrotherapy unless the surgeon recedes permission, or for health or personal preferences of the patient. The hydrotherapy exercises use water resistance, buoyancy and hydrotherapy equipment to achieve the same goals as the land-based therapy. Patients who do not receive hydrotherapy will attend an additional session of land-based physiotherapy, meaning that all patients receive exercise therapy twice a day. Participants will be given a home exercise program to be completed following discharge from inpatient rehabilitation. The home exercise program will be identical to the exercises performed during inpatient rehabilitation. Exercise dosage will be recorded in an exercise diary.

Participants will return to the rehabilitation hospital to participate in the outpatient rehabilitation program approximately one week following discharge from inpatient rehabilitation. All participants will attend once per week for one 45-min session of physiotherapy and either one 45-min session of hydrotherapy (if indicated) or landbased exercise (if hydrotherapy not indicated). These two 45-min sessions will occur consecutively on the same day of each week. Participants will continue their home exercise program and complete their diaries daily.

The exercises in the usual care rehabilitation program are representative of usual care following TKA, and are described in detail in Table 1 and in the Additional file 1. The goals of the usual care rehabilitation program are to improve quadriceps, hamstring and calf strength, to increase knee range of movement and to improve walking and stair climbing ability. Manual therapy including joint mobilization and massage may also be used in addition to exercises to achieve these goals.

\section{Intervention group}

Participants in the intervention group will receive the usual care program of rehabilitation. They will also receive 15 min of additional exercises at each physiotherapy session (total time of $1 \mathrm{~h}$, twice per day) designed specifically to target strengthening of the hip abductor muscles.

The following exercises are unique to the hip strengthening group and have been selected after a review of the literature investigating the efficacy of various hip abductor strengthening exercises (Table 2 and Additional file 1) $[18,19,24-27]$. They will be performed in addition to usual care received following TKA at the rehabilitation hospital. The participants will initially perform non-weight bearing antigravity hip strengthening exercises, such as sidelying hip abduction, prone hip extension, standing abduction. The exercises will be progressed to weight bearing, gravity-resisted exercises such as sideways walking, and hip abduction whilst standing on the operated leg. If the participant receives hydrotherapy they will perform the same additional hip strengthening exercises in the water, to address the identical goals of the land based program.

\section{Control group}

The participants in the control group will receive the usual care program of rehabilitation and will also receive an additional $15 \mathrm{~min}$ of exercise at each physiotherapy session (total session time of $1 \mathrm{~h}$, twice a day) to act as a time control for the additional hip strengthening exercises prescribed for the intervention group (Table 3 and Additional file 1). These exercises have been designed to replicate functional activities such as sit-to-stand, marching and walking around a pre-measured circuit. If the participant receives hydrotherapy they will perform the same additional exercises in the water.

\section{Delivery of the intervention}

Due to the physical nature of the intervention, the treating physiotherapists will not be blinded. Physiotherapists 
Table 1 Usual Care Exercises

\begin{tabular}{ll}
\hline Goal & Exercise \\
\hline Improve quadriceps strength & Static quads \\
& Quads over fulcrum \\
& Forward step-ups \\
& Squats \\
& Leg press \\
& Forward step-downs \\
& Hip and knee flexion \\
Increase active knee flexion ROM & Knee flexion in sitting \\
& Seated slides \\
& Exercise bike \\
& Hamstring curls in prone \\
Improve hamstring strength & Hamstring curls in standing \\
Increase knee extension ROM & Prone hanging \\
Improve calf muscle strength and & Knee extension stretch in sitting \\
flexibility & Heel rises \\
\hline
\end{tabular}

currently employed at the rehabilitation hospital will provide the interventions. The professional qualifications, years of practice and specific training prior to trial initiation will be recorded. All physiotherapists will attend a training session to ensure the exercises are performed accurately and progressed appropriately. At this training session correct and incorrect performance of the exercises will be demonstrated. Exercises will be progressed when able to be completed with minimal fatigue and no significant increase in pain. The physiotherapists will be provided with a detailed manual describing the exercise programs and progression. Follow-up training sessions will be held with the physiotherapists to ensure consistency and address any issues that may arise. Consistency and adherence will also be monitored by the recording of treatment in the participants' medical record. The medical record includes each exercise performed, the number of repetitions of each exercise performed, any variations in the exercises performed and reasons for any variations such as adverse effects.

Table 2 Additional Hip Strengthening Exercises (Intervention Group)

\begin{tabular}{ll}
\hline Goal & Exercise \\
\hline Improve hip strength & Sidelying hip abduction \\
& Prone hip extension \\
& Sideways walking \\
& Standing hip abduction \\
& Hip hitching \\
\hline
\end{tabular}

Table 3 Additional Usual Care Exercises (Control Group)

\begin{tabular}{ll}
\hline Goal & Exercise \\
\hline Improve general function & Sit to stand \\
& Marching \\
& Walking \\
\hline
\end{tabular}

\section{Performance quality}

Performance quality will be monitored by the physiotherapist. The physiotherapist will reassess each participant at the start of each session, and the exercises modified accordingly. If there are significant increases in pain (Visual Analogue Scale $=5$ or greater) the physiotherapist will use his/her clinical reasoning to reduce intensity of particular aggravating exercises or add specific manual physiotherapy treatment as required. To ensure that appropriate muscle adaptation is achieved, the participant's exercises will be progressed according to the American College of Sports Medicine (ACSM) guidelines [28] as soon as the patient is able, and details and criteria for this progression are described in the Additional file 1.

The treating physiotherapist will record the details of each treatment session, and will include the type of exercises and dosage. Any changes to the exercises or adverse responses will be noted. Following discharge from inpatient rehabilitation, participants will be given a home exercise program to continue their exercises and a diary to record daily exercises. The exercises will be progressed during the outpatient phase and the diary used to record dosage and completion of exercises. On discharge from outpatient rehabilitation, the participants will continue to complete the home exercise program until the 6-month follow-up.

\section{Outcome measures}

Demographic information to be collected from all participants includes: name, age, gender, height, weight and body mass index. The type of prosthesis used (including patella resurfacing), left/right TKA, previous joint replacements, co-morbidities, social history (including place of accommodation, occupation/retired), co-interventions (e.g. hydrotherapy), length of hospital stay, number of treatment sessions and analgesics used will be recorded.

The measures that will be used to assess outcome are summarized in Table 4 . The primary outcomes measures are: Knee Injury and Osteoarthritis Outcome score (KOOS) and isometric hip abductor muscle strength. The secondary outcome measures are: Stair Climb Test, 6 min walk test, Timed Up and Go, $40 \mathrm{~m}$ fast-paced walk test, 30-second chair stand test, step test, isometric quadriceps muscle strength, Lower Extremity Functional Scale (LEFS) and SF-12. These physical performance measures were selected based on the recommendations from Osteoarthritis 
Table 4 Outcome measures

\begin{tabular}{|c|c|}
\hline Outcome & Measurement \\
\hline $\begin{array}{l}\text { Knee Injury and Osteoarthritis } \\
\text { Outcome Score }\end{array}$ & $\begin{array}{l}\text { Pain, other symptoms, function } \\
\text { and quality of life }\end{array}$ \\
\hline $\begin{array}{l}\text { Isometric strength of hip } \\
\text { abductors }\end{array}$ & Hand held dynamometer (Newtons) \\
\hline Stair climb test & $\begin{array}{l}\text { Total time (sec) to ascend and } \\
\text { descend } 4 \text { steps. }\end{array}$ \\
\hline 6 min walk test & Distance $(\mathrm{m})$ walked in $6 \mathrm{~min}$ \\
\hline Timed Up and Go & $\begin{array}{l}\text { Timed rise from chair, walk } 3 \mathrm{~m} \\
\text { and return to sitting position. }\end{array}$ \\
\hline $40 \mathrm{~m}$ fast-paced walk test & Time (sec) taken to walk $40 \mathrm{~m}$ \\
\hline 30-second chair stand test & $\begin{array}{l}\text { No. of sit-to-stands performed in } \\
30 \text { seconds }\end{array}$ \\
\hline Step test & $\begin{array}{l}\text { Number of step taps on a step } \\
\text { in } 15 \text { seconds }\end{array}$ \\
\hline Isometric strength of quadriceps & $\begin{array}{l}\text { Hand held dynamometer } \\
\text { (Newtons) }\end{array}$ \\
\hline Knee ROM & Goniometer (degrees) \\
\hline Lower Extremity Functional Scale & Lower extremity functional status \\
\hline SF-12 & Overall Health \\
\hline \multicolumn{2}{|l|}{ Other measures } \\
\hline Compliance & $\begin{array}{l}\text { Therapist treatment records and } \\
\text { patient diary }\end{array}$ \\
\hline Adverse effects & $\begin{array}{l}\text { Therapist treatment records and } \\
\text { patient diary }\end{array}$ \\
\hline Type of TKR & $\begin{array}{l}\text { Operation report or surgeon } \\
\text { contact }\end{array}$ \\
\hline
\end{tabular}

Research Society International (OARSI) for older patients ( $>40$ years old) diagnosed with hip and/or knee OA [29].

Primary and secondary outcomes will be measured at admission to inpatient rehabilitation (approximately 7 days post-operatively), 3 weeks, 6 weeks and 6 months after commencing the rehabilitation program. A discrepancy is often reported in the recovery of patient reported outcomes compared to functional performances, with the more rapid improvement noted in patient reported outcome scales while functional performance measures are often significantly reduced in the early post-operative period [30]. Therefore, the two types of outcome measures have been included to ensure an accurate representation of rehabilitation outcomes following TKA.

\section{Primary outcome measures}

\section{Knee injury and osteoarthritis outcome score (KOOS)}

The Knee Injury and Osteoarthritis Outcome Score (KOOS) is a self report questionnaire with 42-items in 5 separately analyzed subscales of pain, other symptoms, function in daily living, function in sport and recreation, and knee-related quality of life [31]. The tool has good test-retest reliability with $\operatorname{ICC}_{(2,1)}$ values in 26 patients 6 months post TKA of 0.73 (QOL), 0.74 (Sport/recreation),
0.82 (ADL), 0.85 (Symptoms), and 0.94 (Pain). Good content validity demonstrated by $90 \%$ of patients who regarded improvement in the subscales Pain, Symptoms, Activities of Daily Living, and knee-related Quality of Life to be extremely or very important when deciding to have their knee operated on. The KOOS is responsive on all subscales particularly the knee-related Quality of Life subscale (effect size 2.86 at 6 months post TKA and 3.54 at 12 months post TKA) [32].

\section{Isometric strength of hip abductors}

The hip abductors will be tested with the participant in supine with both hips in neutral abduction and rotation. A hand held dynamometer will be used to record force, in Newtons, generated during hip abduction as per the method described previously [33]. The hand-held dynamometer (HHD) will be placed $5 \mathrm{~cm}$ proximal to the lateral femoral condyle. Stabilization of the HHD will be achieved by wrapping a seatbelt around the HHD and securing it to a rail on the adjacent wall at plinth height. The participants will perform a maximal voluntary isometric contraction (MVC) by abducting their limb against the dynamometer and seatbelt, held for 5 seconds. The highest of three consecutive measures will be recorded as the MVC. This method of testing hip abductor strength was reliable and valid in a sample of patients similar to those intended for the current study [33]. The seatbelt will be used to resist hip abductor strength, as this method is less susceptible to the individual strength of the therapist [34].

\section{Secondary outcome measures Stair climb test}

The stair climb test has been used extensively as an outcome measure in TKA [3, 13, 35-37]. The test assesses the time, in seconds, to ascend and descend a flight of stairs. The number of stairs in the flight and the step height are recorded. In the current study, a four-step staircase will be used. The use of a handrail and gait aids are permitted and will be recorded, as will be the footstep pattern (reciprocal or step-to). Inter-rater reliability was reported as $\operatorname{ICC}_{(2,1)}=0.94$ (95\% CI =0.55-0.98). MDC $_{90}$ was calculated as 2.6 seconds [38]. The stair climb test has demonstrated adequate construct validity [39] and responsiveness detecting initial deterioration (effect size $=-0.84$ ) from pre-op to 1 month post-TKA scores, and then subsequent improvement (effect size $=1.26$ ) from 1 month to 12 months post-TKA on a 12-step flight [36].

\section{Minute Walk Test (6 MWT)}

The 6 min walk test (6MWT) is a frequently used measure for patients following TKA [3, 30, 35, 37, 40-45]. It is a test of aerobic capacity and long distance walking ability [29]. Patients are instructed to walk with usual gait aids on a premeasured circuit, covering as much 
distance as possible during the 6 min time frame. Rests are permitted and are included in the time. Test-retest reliability in participants with end-stage hip and knee OA awaiting joint replacement was calculated as $\operatorname{ICC}_{(2,1)}=0.94$ (95\% CI $=0.88-0.98)$ with standard error of measurement (SEM) of $26.3 \mathrm{~m}$ and $\mathrm{MDC}_{90}$ of $61.34 \mathrm{~m}$ [38]. The 6MWT is responsive when evaluating early recovery two to four months post TKA (effect size $=0.82, \mathrm{SRM}=1.51$ ) [30].

\section{Timed up and Go Test (TUG)}

The timed up and go test (TUG) measures the time, in seconds, that a patient takes to stand from an armed chair, walk for $3 \mathrm{~m}$, and return to sit on the same chair. A walking aid can be used if required [46]. The reliability of the TUG has been established in a study that combined pre-operative TKA and THA subjects. The test-retest reliability was reported as $\operatorname{ICC}_{(2,1)}=0.75(95 \% \mathrm{CI}=0.51$ 0.98) [38]. The TUG was found to be selective in its ability to distinguish healthy subjects from TKA patients [47]. It is responsive to detecting early deterioration and improvement in the early post-operative period with standardized response means (SRM) varying from -1.08 (95\% CI -1.38 to -0.92$)$, indicating a worsening in the value to 1.04 (95\% CI 0.84 to 1.61 ) indicating an improvement in time taken to perform the test. Standard error of measurement (SEM) was calculated as 1.07 (95\% CI $=0.86-1.41)$ and minimal detectable change at the $90 \%$ confidence level $\left(\mathrm{MDC}_{90}\right)$ was 2.49 seconds [38].

\section{0 m Fast-paced Walk test}

The purpose of the $40 \mathrm{~m}$ fast-paced walk test is to assess short distance walking ability [29]. The test measures the time, in seconds, taken to walk $40 \mathrm{~m}$ as quickly but as safely as possible excluding turns. The total time is expressed as $\mathrm{m} / \mathrm{s}$ by dividing distance $(40 \mathrm{~m})$ by time (seconds). Any gait aids required are recorded [48]. The reliability and validity of the $40 \mathrm{~m}$ fast-paced walk test has been tested in a group of combined pre-operative THA and TKA patients, where test-retest reliability was found to be excellent, $\mathrm{ICC}_{(2,1)}=0.91(95 \% \mathrm{CI}=0.81-0.97)$ and responsiveness to initial surgery followed by recovery was evident [38] Standard error of measurement (SEM) was calculated as $1.73(95 \% \mathrm{CI}=1.39-2.29)$ and minimal detectable change at the $90 \%$ confidence level $\left(\mathrm{MDC}_{90}\right)$ was 4.04 sec [38].

\section{0-second chair stand test}

The 30-second chair stand test (30s CST) is a test of sitto-stand ability, lower limb strength and dynamic balance [29]. Starting from a seated position, the patient stands then sits as many times as possible in 30 seconds [49]. The chair is a straight back chair with a $44 \mathrm{~cm}$ seat height, preferably without arms. If the patient cannot stand without using their arms, their hands can be placed on their legs and recorded as an adapted test score [29]. An initial practice trial will be performed to reduce any practice effect [50]. In a population with end stage hip or knee OA awaiting joint replacement surgery, intra-tester reliability $\left(\operatorname{ICC}_{(1,1)}=0.97-0.98,95 \% \mathrm{CI}=0.94-0.99\right)$ and inter-tester reliability $\left(\mathrm{ICC}_{(1,1)} 0.93-0.98,95 \% \mathrm{CI}=0.87\right.$ 0.99 ) were excellent [50]. Convergent validity was evident by a moderate correlation (Spearman's rho $=0.64$ ( $95 \% \mathrm{CI}$ 0.49 to 0.75$)$ ) with other measures of physical function. Discriminant validity was evident by low correlation (Spearman's rho $=0.33$ (95\% CI 0.12 to 0.51$)$ ) with mental health scores. Known groups validity was demonstrated by significantly higher scores recorded for participants who ambulated without a gait aid compared to participants who did not with a mean difference of 2.8 (95\% CI 1.4 to 4.1 ) and effect size of 0.64 (95 \% CI 0.32 to 0.95 ). Standardized response mean of 0.84 (95\% CI 0.61 to 1.07) indicates the 30s CST is a responsive measure [51].

\section{Step test}

The step test is a functional, dynamic test of standing balance [52] which has been commonly used to measure outcome of interventions in patients with OA knee [53-57]. This test is used to assess the ability of the patient to maintain standing balance whilst performing a potentially destabilizing movement [58]. The step test involves stepping one foot on, then off, a block as quickly as possible in 15 seconds without holding a support. The same procedure is then repeated with the opposite leg stepping [52]. It has high test-retest reliability in healthy older subjects (ICC $>0.90$ ), good concurrent validity and is sensitive to changes in performance over time [52].

\section{Isometric strength quadriceps muscle}

Isometric quadriceps strength will be measured against a strap and HHD with the patient sitting and the knee at $90^{\circ}$ flexion [59]. Isometric quadriceps strength has been tested at knee flexion angles of $45-90^{\circ}$ [60] and is recommended to be tested at $60-90^{\circ}$ knee flexion [61] Isometric quadriceps strength measured with HHD at $90^{\circ}$ knee flexion has demonstrated excellent reliability $\left.\operatorname{ICC}_{(2,1)}=0.96\right)$ and $\mathrm{MDC}_{95}$ of $0.58 \mathrm{~N} / \mathrm{kg}$ following TKA [59]. If the participant cannot flex their knee to $90^{\circ}$, isometric quadriceps strength will be measured at the maximum range of flexion available and the range will be recorded. The hand-held dynamometer (HHD) will be placed on the anterior aspect of the distal tibia, $5 \mathrm{~cm}$ proximal to medial malleolus. Stabilization of the HHD will be achieved by wrapping a seatbelt around the HHD and securing it to a fixed attachment of the plinth. The participants will perform a maximal voluntary isometric contraction (MVC) by extending their lower leg against the dynamometer and seatbelt, held for 5 seconds. 
The highest of three consecutive measures will be recorded as the MVC.

\section{Passive knee range of movement (PROM)}

Passive knee ROM will be measured with a goniometer axis placed at the lateral epicondyle of the femur, the proximal arm parallel to the long axis of the femur \& pointing at the greater trochanter, the distal arm parallel to the long axis of the fibula and pointing at the lateral malleolus. Maximum passive flexion and maximum passive extension range of movement will be recorded.

\section{Lower Extremity Functional Scale (LEFS)}

The Lower Extremity Functional Scale (LEFS) is a 20item self-report measure of lower extremity functional status. Each item is scored on a 5-point scale (0-4) and the total LEFS scores ranges from 0 to 80 with higher scores reflecting greater levels of functional status [62]. Test-retest reliability has been reported as excellent $\left(\operatorname{ICC}_{(2,1)}=0.85\right), \mathrm{SEM}=3.7$ points and $\mathrm{MDC}_{90}=9$ points in a combined group TKA and THA [43, 63-66]. Internal consistency was reported as 0.93 while cross sectional validity $(r=0.68)$ and longitudinal validity $(r=0.64)$ were also good [63].

\section{SF-12}

The SF-12 is a generic measure of a patient's general physical and mental well-being [67] based on the SF-36 score [68]. Patients' general and physical wellbeing can influence the outcome of joint-specific scores following TKA [69]. The SF-12 has been used extensively to measure outcomes following TKA [69-75]. Reliability coefficients of 0.84 and 0.80 were observed for the physical component and mental components respectively in a pre-operative TKA population [76]. The level of patient satisfaction with TKA patients' pain relief and function correlated with the improvement in the physical component of the SF-12 score $(\mathrm{r}=0.51, P<0.001$ and $\mathrm{r}=0.60$, $P<0.001)$ respectively. The minimal clinically important difference (MCID) for the physical component of the SF-12 was 4.5 points (95\% CI $=3.9$ to 5.2 ) and 4.8 points ( $95 \% \mathrm{CI}=4.2$ to 5.4 ) for pain relief and function component respectively [77].

\section{Other measures \\ Compliance}

Compliance will be assessed by review of the patient notes recorded by the treating physiotherapist. These notes will be assessed for the type of exercise, dosage of exercises and adverse events for each treatment session. On discharge from inpatient rehabilitation patients will be given an exercise diary, which will record the dosage of each exercise and any adverse events experienced daily.

\section{Adverse effects}

Any adverse responses will be noted in the patient notes located in the patient's hospital file. The physiotherapists will be trained to recognize adverse events such as significant increases in knee pain, lateral hip pain in the training session held prior to the commencement of the trial.

\section{Type of TKA}

Details will be taken from operation report or contact with the surgeon if not recorded on operation report. The surgical approach, presence of patellar resurfacing, type of prosthesis including cruciate retaining, posterior stabilized or other, will be recorded.

\section{Data and statistical analysis}

An estimate of sample size was made on the ability to detect a medium effect (Cohen's $f=0.25$ ). The assumption of obtaining a medium effect size was reasonable based on the effect sizes reported for similar measures such as the WOMAC by other studies in the literature (effect size for WOMAC function $=0.3$, WOMAC pain $=0.31$ ) $[18]$. A standard statistical package was used [78]. To obtain a power of 0.8 at a significance level of $\alpha=0.05$, a minimum sample of 82 participants would be needed. To allow for loss to follow-up the aim was to recruit a sample of approximately 104 participants.

Participant characteristics and baseline data will be summarized by descriptive statistics. Independent sample $\mathrm{t}$-tests for parametric data (or a chi-squared test for categorical data) will be used to determine the comparability of the two groups at baseline on measures of age, gender and BMI. All data will be analysed using intention to treat principles. Any missing data will be replaced by the last observation carried forward. Repeated measures ANOVA (group by time) calculations will be used to determine the main effects and interactions of group (hip abductor strengthening vs usual care exercises) and time (repeated factor: baseline, 3 weeks, 6 weeks, 6 months) for all outcome measures. Statistical analysis will be completed using SPSS software (version 22) and the significance level set at $P<0.05$.

\section{Discussion}

This study describes a protocol for a RCT that will investigate whether the addition of hip abductor muscle strengthening to the usual care rehabilitation program following TKA leads to superior performance based and patient reported outcomes. This is the first reported study to specifically assess the effect of hip strengthening exercises in this population. The trial will provide information to clinicians to potentially improve outcomes for patients with TKA. This is particularly relevant as the number of patients undergoing TKA is rapidly increasing worldwide [79-81]. 
Many studies striving to achieve better outcomes for TKA patients continue to focus on impairments associated directly with the knee joint. Recovery has been variable and the majority of patients continue to demonstrate lower extremity muscle weakness and functional deficits such as slower walking speeds, difficulty negotiating stairs and difficulty rising from a chair when compared to agematched healthy individuals [13, 60, 82-84]. Therefore, it is reasonable to hypothesize that the causes of disability and poor function following TKA may also be related to other joints, particularly the hip. This hypothesis is further strengthened by the presence of hip abductor muscle weakness in patients with knee OA [14] which persists post TKA [20]. Patients with greater lower limb strength following TKA perform better on functional activities [83] demonstrating that lower limb strength contributes to functional performance. Investigations into the role of hip abductor strength following TKA have showed significant contributions of hip strength to function [21]. This is not surprising given that hip strength and hip joint mechanics have a close relationship to normal knee function. Achieving optimal outcomes following TKA therefore would require optimal hip strength in combination with optimal quadriceps strength. The investigation into targeted hip strengthening is warranted in this patient group.

It is important to evaluate the effectiveness of rehabilitation programs with appropriate outcome measures. This trial has ensured that outcome measures are included from the three different domains of physical impairment, patient reported measures, and physical performance measures, as recommended by OARSI and Mizner et al. [29, 36]. This is advantageous because many of the outcome measures for the different domains improve at different rates postoperatively. In the early stages after TKA surgery scores on the patient reported outcome scales are significantly better than preoperative scores, while functional performance outcome measures are reduced [36]. This may be due to the experience of pain free movement combined with the ability to perform a previously painful and difficult activity again resulting in high patient satisfaction and is reflected in the high scores of patient reported outcome scales [85]. The performance-based tests demonstrate an initial reduction in physical function in patients following TKA that is not seen in patient-reported measures [36]. From 1 month to 6 months postoperatively, patients' physical performance improves $[3,43,83]$ with the highest rate of improvement occurring in the period from 1 to 3 months compared with the period from 3 to 6 months $[43,35]$. The outcome measures included in this trial ensure an accurate picture of recovery will be recorded.

In the evaluation of the effects of hip strengthening following TKA, it is important to determine that hip strengthening has occurred as a result of the intervention.
The method of strength measurement may be considered a limitation of the study however the use of a hand-held dynamometer rather than a mechanical isokinetic dynamometer is reliable and valid with a seatbelt for restraint [33]. As this study will be conducted during the participants' rehabilitation in a small hospital, access to an isokinetic dynamometer is not possible.

A second limitation of the study is a lack of pre-operative testing of hip abductor strength as well as other outcome measures, which would add value to the study. However, the patients that require inpatient rehabilitation in this study are not identified pre-operatively. The need for inpatient rehabilitation is identified post-operatively on an individual basis and outpatient rehabilitation is determined on arrival to the rehabilitation hospital. The lack of pre-operative testing is taken into account by measuring the hip abductor and quadriceps muscle strength at all time points in the study. The recovery of muscle strength in both groups can then be compared and their contribution to functional gains calculated.

The exercise programs described are standardized by guidelines for progression according to the American College of Sports Medicine (ACSM) guidelines [28]. Progression of exercises will also be based on the physiotherapists' clinical assessment and tailored to the individual participants needs. The usual care exercise program has additional functional exercises to account for the extra time spent on hip strengthening exercises by the hip strengthening group participants. The use of additional manual therapy will also be decided for individual participants according to their clinical need.

There is a need for more knowledge regarding specific hip strengthening compared to usual care following TKA. The findings of this study will guide clinicians regarding the use of hip strengthening exercises in TKA rehabilitation programs.

\section{Additional file}

Additional file 1: Exercise description and progression. Table S1. Usual care exercises. Table S2. Additional usual care exercises. Table S3. Additional Hip exercises. (DOCX 53194 kb)

\footnotetext{
Abbreviations

30s CST, 30-second chair stand test; 6MWT, 6 min walk test; $95 \% \mathrm{Cl}, 95 \%$ confidence interval; ACSM, American College of sports medicine; ADL, activities of daily living; ANOVA, analysis of variance; BMI, body mass index; CONSORT, consolidated standards of reporting trials; HHD, hand held dynamometer; ICC, intra-class coefficient; KOOS, knee injury and osteoarthritis outcome score; LEFS, lower extremity functional scale; $\mathrm{m} / \mathrm{s}$, metres per second; $\mathrm{MDC}_{90}$, minimal detectable change at the $90 \%$ confidence level; MVC, maximal voluntary contraction; OA, osteoarthritis; OARSI, osteoarthritis research society international; $\mathrm{QOL}$, quality of life; RCT, randomized controlled trial; ROM, range of movement; SCT, stair climb test; SEM, standard error of measurement; SPIRIT, standard protocol items: recommendation for interventional trials; TKA, total knee arthroplasty; TUG, timed up and go test; WOMAC, Western Ontario and McMaster Universities arthritis index
} 


\section{Acknowledgements}

Not applicable.

\section{Funding}

The study has not received any external funding.

\section{Availability of data and materials}

No data are presented, as this is a study protocol.

\section{Authors' contributions}

MS, JM, KW have participated in the design of the study, and help to draft the manuscript. MS will collect the data. All authors read and approved the final manuscript and will contribute to the analysis and interpretation of the data.

\section{Competing interests}

The authors declare that they have no competing interests.

\section{Consent to publish}

The subject whose images appear in the Additional file 1 has provided consent for his images to be used.

\section{Ethics and consent to participate}

Ethical approval was granted by La Trobe University Faculty Human Ethics Committee (FHEC 14/256). Subjects will give their written informed consent to participate.

\section{Received: 7 December 2015 Accepted: 27 May 2016}

\section{Published online: 13 June 2016}

\section{References}

1. Page CJ, Hinman RS, Bennell KL. Physiotherapy management of knee osteoarthritis. Int J Rheum Dis. 2011;14(2):145-51.

2. Peat G, McCarney R, Croft P. Knee pain and osteoarthritis in older adults: a review of community burden and current use of primary health care. Ann Rheum Dis. 2001:60(2):91-7.

3. Bade MJ, Kohrt WM, Stevens-Lapsley JE. Outcomes before and after total knee arthroplasty compared to healthy adults. J Orthop Sports Phys Ther. 2010;40(9):559-67.

4. Levine M, McElroy K, Stakich V, Cicco J. Comparing conventional physical therapy rehabilitation with neuromuscular electrical stimulation after TKA. Orthopedics. 2013;36(3):e319-24.

5. Petterson SC, Mizner RL, Stevens JE, Raisis L, Bodenstab A, Newcomb W, et al. Improved function from progressive strengthening interventions after total knee arthroplasty: a randomized clinical trial with an imbedded prospective cohort. Arthritis Rheum. 2009;61(2):174-83.

6. Piva SR, Almeida G, Gil AB, Teixeira PE, Fitzgerald GK. Effectiveness and feasibility of a balance training program post total knee arthroplasty: pilot randomized trial. J Orthop Sports Phys Ther. 2009:39(1):A29-30.

7. Meier W, Mizner R, Marcus R, Dibble L, Peters C, Lastayo PC. Total knee arthroplasty: muscle impairments, functional limitations, and recommended rehabilitation approaches. J Orthop Sports Phys Ther. 2008;38(5):246-56.

8. Minns Lowe CJ, Barker KL, Dewey ME, Sackley CM. Effectiveness of physiotherapy exercise following hip arthroplasty for osteoarthritis: a systematic review of clinical trials. 2009.

9. Ranawat CS, Ranawat AS, Mehta A. Total knee arthroplasty rehabilitation protocol: what makes the difference? J Arthroplasty. 2003;18(3 Suppl 1):27-30

10. Westby MD, Backman CL. Patient and health professional views on rehabilitation practices and outcomes following total hip and knee arthroplasty for osteoarthritis:a focus group study. BMC Health Serv Res. 2010;10:119.

11. Bade M, Stevens-Lapsley JE. Restoration of physical function in patients following total knee arthroplasty: an update on rehabilitation practices. Curr Opin Rheumatol. 2012;24(2):208-14.

12. Mizner RL, Snyder-Mackler L. Altered loading during walking and sit-to-stand is affected by quadriceps weakness after total knee arthroplasty. J Orthop Res. 2005:23(5):1083-90.

13. Walsh M, Woodhouse LJ, Thomas SG, Finch E. Physical impairments and functional limitations: a comparison of individuals 1 year after total knee arthroplasty with control subjects. Phys Ther. 1998;78(3):248-58.
14. Hinman RS, Hunt MA, Creaby MW, Wrigley TV, McManus FJ, Bennell KL. Hip muscle weakness in individuals with medial knee osteoarthritis. Arthritis Care Res. 2010;62(8):1190-3.

15. Childs JD, Sparto PJ, Fitzgerald GK, Bizzini M, Irrgang JJ. Alterations in lower extremity movement and muscle activation patterns in individuals with knee osteoarthritis. Clin Biomech. 2004;19(1):44-9.

16. Al-Zahrani KS, Bakheit AM. A study of the gait characteristics of patients with chronic osteoarthritis of the knee. Disabil Rehabil. 2002;24(5):275-80.

17. Farr JN, Going SB, Lohman TG, Rankin L, Kasle S, Cornett M, et al. Physical activity levels in patients with early knee osteoarthritis measured by accelerometry. Arthritis Rheum. 2008;59(9):1229-36.

18. Bennell KL, Hunt MA, Wrigley TV, Hunter DJ, McManus FJ, Hodges PW, et al. Hip strengthening reduces symptoms but not knee load in people with medial knee osteoarthritis and varus malalignment: a randomised controlled trial. Osteoarthr Cartil. 2010;18(5):621-8.

19. Sled EA, Khoja L, Deluzio KJ, Olney SJ, Culham EG. Effect of a home program of hip abductor exercises on knee joint loading, strength, function, and pain in people with knee osteoarthritis: a clinical trial. Phys Ther. 2010;90(6):895-904.

20. Alnahdi AH, Zeni JA, Snyder-Mackler L. Hip abductor strength reliability and association with physical function after unilateral total knee arthroplasty: a cross-sectional study. Phys Ther. 2014;94(8):1154-62.

21. Piva SR, Teixeira PE, Almeida GJ, Gil AB, DiGioia III AM, Levison TJ, et al. Contribution of hip abductor strength to physical function in patients with total knee arthroplasty. Phys Ther. 2011;91(2):225-33.

22. Chan AW, Tetzlaff JM, Altman DG, Laupacis A, Gotzsche PC, Krleza-Jeric K, et al. SPIRIT 2013 statement: defining standard protocol items for clinical trials. Ann Intern Med. 2013;158(3):200-7.

23. Boutron I, Moher D, Altman DG, Schulz KF, Ravaud P, Group C. Extending the CONSORT statement to randomized trials of nonpharmacologic treatment: explanation and elaboration. Ann Intern Med. 2008:148(4):295-309.

24. Bolgla LA, Uhl TL. Electromyographic analysis of hip rehabilitation exercises in a group of healthy subjects. J Orthop Sports Phys Ther. 2005:35(8):487-94.

25. Ekstrom RA, Donatelli RA, Carp KC. Electromyographic analysis of core trunk, hip, and thigh muscles during 9 rehabilitation exercises. J Orthop Sports Phys Ther. 2007;37(12):754-62.

26. Jacobs CA, Lewis $M$, Bolgla LA, Christensen CP, Nitz AJ, Uhl TL. Electromyographic analysis of hip abductor exercises performed by a sample of total hip arthroplasty patients. J Arthroplasty. 2009:24(7):1130-6.

27. Thorborg K, Bandholm T, Petersen J, Weeke KMO, Weinold C, Andersen B, et al. Hip abduction strength training in the clinical setting: With or without external loading? Scand J Med Sci Sports. 2010;20(2):70-7.

28. Garber CE, Blissmer B, Deschenes MR, Franklin BA, Lamonte MJ, Lee IM, et al. American College of Sports Medicine position stand. Quantity and quality of exercise for developing and maintaining cardiorespiratory, musculoskeletal, and neuromotor fitness in apparently healthy adults: guidance for prescribing exercise. Med Sci Sports Exerc. 2011;43(7):1334-59.

29. Dobson F, Hinman RS, Roos EM, Abbott JH, Stratford P, Davis AM, et al. OARSI recommended performance-based tests to assess physical function in people diagnosed with hip or knee osteoarthritis. Osteoarthr Cartil. 2013;21(8):1042-52.

30. Parent $\mathrm{E}$, Moffet $\mathrm{H}$. Comparative responsiveness of locomotor tests and questionnaires used to follow early recovery after total knee arthroplasty. Arch Phys Med Rehabil. 2002;83(1):70-80.

31. Peer MA, Lane J. The Knee Injury and Osteoarthritis Outcome Score (KOOS): A Review of Its Psychometric Properties in People Undergoing Total Knee Arthroplasty. J Orthop Sports Phys Ther. 2013:43(1):20-8.

32. Roos EM, Toksvig-Larsen S. Knee injury and Osteoarthritis Outcome Score (KOOS) - validation and comparison to the WOMAC in total knee replacement. Health Qual Life Outcomes. 2003;1:17.

33. Schache MB, McClelland JA, Webster KE. Reliability of measuring hip abductor strength following total knee arthroplasty using a hand-held dynamometer. Disabil Rehabil. 2015;20:1-4.

34. Katoh M, Yamasaki H. Comparison of reliability of isometric leg muscle strength measurements made using a hand-held dynamometer with and without a restraining belt. J Phys Ther Sci. 2009:21(1):37-42.

35. Farquhar S, Snyder-Mackler L. The Chitranjan Ranawat Award: The nonoperated knee predicts function 3 years after unilateral total knee arthroplasty. Clin Orthop Relat Res. 2010;468(1):37-44. 20090527.

36. Mizner RL, Petterson SC, Clements KE, Zeni Jr JA, Irrgang JJ, Snyder-Mackler L. Measuring functional improvement after total knee arthroplasty requires both performance-based and patient-report assessments: a longitudinal analysis of outcomes. J Arthroplasty. 2011;26(5):728-37. 
37. Yoshida Y, Mizner RL, Ramsey DK, Snyder-Mackler L. Examining outcomes from total knee arthroplasty and the relationship between quadriceps strength and knee function over time. Clin Biomech. 2008;23(3):320-8.

38. Kennedy DM, Stratford PW, Wessel J, Gollish JD, Penney D. Assessing stability and change of four performance measures: a longitudinal study evaluating outcome following total hip and knee arthroplasty. BMC Musculoskelet Disord. 2005;6:3.

39. Almeida GJ, Schroeder CA, Gil AB, Fitzgerald GK, Piva SR. Interrater reliability and validity of the stair ascend/descend test in subjects with total knee arthroplasty. Arch Phys Med Rehabil. 2010;91(6):932-8.

40. Bruun-Olsen V, Heiberg KE, Wahl AK, Mengshoel AM. The immediate and long-term effects of a walking-skill program compared to usual physiotherapy care in patients who have undergone total knee arthroplasty (TKA): a randomized controlled trial. Disabil Rehabil. 2013;35(23):2008-15.

41. Choi S, Trang A, McCartney CJ. Reporting functional outcome after knee arthroplasty and regional anesthesia: a methodological primer. Reg Anesth Pain Med. 2013;38(4):340-9.

42. Jakobsen $\mathrm{TL}$, Kehlet $\mathrm{H}$, Husted $\mathrm{H}$, Petersen J, Bandholm T. Early progressive strength training to enhance recovery after fast-track total knee arthroplasty: a randomized controlled trial. Arthritis Care Res. 2014;66(12):1856-66.

43. Kennedy DM, Stratford PW, Riddle DL, Hanna SE, Gollish JD. Assessing recovery and establishing prognosis following total knee arthroplasty. Phys Ther. 2008;88(1):22-32.

44. Naylor JM, Crosbie J, Ko V. Is there a role for rehabilitation streaming following total knee arthroplasty? Preliminary insights from a randomized controlled trial. J Rehabil Med. 2015;47(3):235-41.

45. Su EP, Perna M, Boettner F, Mayman DJ, Gerlinger T, Barsoum W, et al. A prospective, multi-center, randomised trial to evaluate the efficacy of a cryopneumatic device on total knee arthroplasty recovery. J Bone Joint Surg (Br). 2012;94(11):153-6.

46. Podsiadlo D, Richardson S. The timed "Up \& Go": a test of basic functional mobility for frail elderly persons. J Am Geriatr Soc. 1991;39(2):142-8.

47. Boonstra MC, De Waal Malefijt MC, Verdonschot N. How to quantify knee function after total knee arthroplasty? Knee. 2008;15(5):390-5.

48. Wright AA, Cook CE, Baxter GD, Dockerty JD, Abbott JH. A comparison of 3 methodological approaches to defining major clinically important improvement of 4 performance measures in patients with hip osteoarthritis. J Orthop Sports Phys Ther. 2011;41(5):319-27.

49. Jones CJ, Rikli RE, Beam WC. A 30-s chair-stand test as a measure of lower body strength in community-residing older adults. Res Q Exerc Sport. 1999;70(2):113-9.

50. Gill S, McBurney H. Reliability of performance-based measures in people awaiting joint replacement surgery of the hip or knee. Physiother Res Int. 2008;13(3):141-52.

51. Gill SD, De Morton NA, Mc BH. An investigation of the validity of six measures of physical function in people awaiting joint replacement surgery of the hip or knee. Clin Rehabil. 2012;26(10):945-51.

52. Hill KD, Bernhardt J, McGann AM, Maltese D, Berkovits D. A new test of dynamic standing balance for stroke patients: reliability, validity and comparison with healthy elderly. Physiother Can. 1996;48(4):257-62.

53. Coleman S, McQuade J, Rose J, Inderjeeth C, Carroll G, Briffa NK. Selfmanagement for osteoarthritis of the knee: does mode of delivery influence outcome? BMC Musculoskelet Disord. 2010;11:56.

54. Goncalves RS, Pinheiro JP, Cabri J. Evaluation of potentially modifiable physical factors as predictors of health status in knee osteoarthritis patients referred for physical therapy. Knee. 2012;19(4):373-9.

55. Hale LA, Waters D, Herbison P. A randomized controlled trial to investigate the effects of water-based exercise to improve falls risk and physical function in older adults with lower-extremity osteoarthritis. Arch Phys Med Rehabil. 2012;93(1):27-34.

56. Hinman RS, Bennell KL, Crossley KM, McConnell J. Immediate effects of adhesive tape on pain and disability in individuals with knee osteoarthritis. Rheumatology (Oxford). 2003:42(7):865-9.

57. Lim BW, Hinman RS, Wrigley TV, Sharma L, Bennell KL. Does knee malalignment mediate the effects of quadriceps strengthening on knee adduction moment, pain, and function in medial knee osteoarthritis? A randomized controlled trial. Arthritis Rheum. 2008;59(7):943-51.

58. Hinman RS, Bennell KL, Metcalf BR, Crossley KM. Balance impairments in individuals with symptomatic knee osteoarthritis: a comparison with matched controls using clinical tests. Rheumatology (Oxford). 2002; 41(12):1388-94
59. Koblbauer IF, Lambrecht $\mathrm{Y}$, van der Hulst ML, Neeter C, Engelbert RH, Poolman RW, et al. Reliability of maximal isometric knee strength testing with modified hand-held dynamometry in patients awaiting total knee arthroplasty: useful in research and individual patient settings? A reliability study. BMC Musculoskelet Disord. 2011;12:249.

60. Schache MB, McClelland JA, Webster KE. Lower limb strength following total knee arthroplasty: a systematic review. Knee. 2014;21(1):12-20.

61. Alnahdi $\mathrm{AH}$. Outcome measures capturing ICF domains in patient with total knee arthroplasty. Int J Rehabil Res. 2014;37(4):281-9.

62. Binkley JM, Stratford PW, Lott SA, Riddle DL. The Lower Extremity Functional Scale (LEFS): scale development, measurement properties, and clinical application. North American Orthopaedic Rehabilitation Research Network. Phys Ther. 1999;79(4):371-83.

63. Stratford PW, Binkley JM, Watson J, Heath-Jones T. Validation of the LEFS on patients with total joint arthroplasty. Physiother Can. 2000;52(2):97.

64. Stratford PW, Kennedy DM. Performance measures were necessary to obtain a complete picture of osteoarthritic patients. J Clin Epidemiol. 2006;59(2):160-7.

65. Stratford PW, Kennedy DM, Hanna SE. Condition-specific Western Ontario McMaster Osteoarthritis Index was not superior to region-specific Lower Extremity Functional Scale at detecting change. J Clin Epidemiol. 2004;57(10):1025-32.

66. Stratford PW, Kennedy DM, Maly MR, Macintyre NJ. Quantifying self-report measures' overestimation of mobility scores postarthroplasty. Phys Ther. 2010;90(9):1288-96.

67. Ware Jr J, Kosinski M, Keller SD. A 12-Item Short-Form Health Survey: construction of scales and preliminary tests of reliability and validity. Med Care. 1996;34(3):220-33.

68. Ware Jr JE, Sherbourne CD, The MOS. 36-item short-form health survey (SF-36). I. Conceptual framework and item selection. Med Care. 1992;30(6):473-83.

69. Clement ND, Burnett R. Patient satisfaction after total knee arthroplasty is affected by their general physical well-being. Knee Surg Sports Traumatol Arthrosc. 2013;21(11):2638-46.

70. Blakeney WG, Khan RJ, Palmer JL. Functional outcomes following total knee arthroplasty: a randomised trial comparing computer-assisted surgery with conventional techniques. Knee. 2014;21(2):364-8.

71. Dowsey MM, Nikpour M, Choong PF. Outcomes following large joint arthroplasty: does socio-economic status matter? BMC Musculoskelet Disord. 2014;15:148

72. Hamilton D, Henderson GR, Gaston P, MacDonald D, Howie C, Simpson AH. Comparative outcomes of total hip and knee arthroplasty: a prospective cohort study. Postgrad Med J. 2012;88(1045):627-31.

73. Maniar RN, Baviskar JV, Singhi T, Rathi SS. To use or not to use continuous passive motion post-total knee arthroplasty presenting functional assessment results in early recovery. J Arthroplasty. 2012;27(2):193-200.e1.

74. Mockford BJ, Thompson NW, Humphreys P, Beverland DE. Does a standard outpatient physiotherapy regime improve the range of knee motion after primary total knee arthroplasty? J Arthroplasty. 2008;23(8):1110-4.

75. Noticewala MS, Geller JA, Lee JH, Macaulay W. Unicompartmental knee arthroplasty relieves pain and improves function more than total knee arthroplasty. J Arthroplasty. 2012;27(8 Suppl):99-105.

76. Poitras S, Beaule PE, Dervin GF. Validity of A Short-Term Quality of Life Questionnaire in Patients Undergoing Joint Replacement: The Quality of Recovery-40. J Arthroplast. 2012;27(9):1604-8.e1.

77. Clement ND, MacDonald D, Simpson AH. The minimal clinically important difference in the Oxford knee score and Short Form 12 score after total knee arthroplasty. Knee Surg Sports Traumatol Arthrosc. 2014;22(8):1933-9.

78. Faul F, Erdfelder E, Lang AG, Buchner A. G Power 3: a flexible statistical power analysis program for the social, behavioral, and biomedical sciences. Behav Res Methods. 2007;39(2):175-91.

79. Australian Orthopaedic Association. National Joint Replacement Registry. Annual Report. Adelaide: AOA; 2015.

80. Kurtz S, Ong K, Lau E, Mowat F, Halpern M. Projections of primary and revision hip and knee arthroplasty in the United States from 2005 to 2030. J Bone Joint Surg Am. 2007;89(4):780-5.

81. Kurtz SM, Ong KL, Lau E, Widmer M, Maravic M, Gomez-Barrena E, et al. International survey of primary and revision total knee replacement. Int Orthop. 2011;35(12):1783-9.

82. McClelland JA, Webster KE, Feller JA. Gait analysis of patients following total knee replacement: a systematic review. Knee. 2007;14(4):253-63.

83. Mizner RL, Petterson SC, Snyder-Mackler L. Quadriceps strength and the time course of functional recovery after total knee arthroplasty. J Orthop Sports Phys Ther. 2005;35(7):424-36. 
84. Rossi MD, Hasson S, Kohia M, Pineda E, Bryan W. Mobility and perceived function after total knee arthroplasty. J Arthroplasty. 2006;21(1):6-12.

85. Senden R, Grimm B, Meijer K, Savelberg H, Heyligers IC. The importance to including objective functional outcomes in the clinical follow up of total knee arthroplasty patients. Knee. 2011;18(5):306-11.

Submit your next manuscript to BioMed Central and we will help you at every step:

- We accept pre-submission inquiries

- Our selector tool helps you to find the most relevant journal

- We provide round the clock customer support

- Convenient online submission

- Thorough peer review

- Inclusion in PubMed and all major indexing services

- Maximum visibility for your research

Submit your manuscript at www.biomedcentral.com/submit 